\title{
A point mutation in the zinc finger motif of RID1/EHD2/OsID1 protein leads to outstanding yield-related traits in japonica rice variety Wuyunjing 7
}

Shikai Hư ${ }^{\dagger}$, Guojun Dong ${ }^{\dagger}$, Jie Xu, Yan Su, Zhenyuan Shi, Weijun Ye, Yuanyuan Li, Gengmi Li, Bin Zhang, Jiang Hu, Qian Qian, Dali Zeng ${ }^{*}$ and Longbiao Guo*

\begin{abstract}
Background: Flowering time, which is often associated with the length of the growth period in rice, determines the adaptability of a plant to various environments. However, little is known about how flowering-time genes affect panicle development and yield formation potential in rice after inducing the transition from vegetative growth to reproductive growth.

Results: To explore the relationship between floral induction and yield formation and the molecular mechanism of panicle development in rice, a novel mutant, ghd10, was identified from japonica variety Wuyunjing 7 plants subjected to ethyl methane sulfonate (EMS) treatment. The ghd10 mutant exhibited delayed flowering time, tall stalks and increased panicle length and primary branch number. Map-based cloning revealed that Ghd10 encodes a transcription factor with Cys-2/His-2-type zinc finger motifs. Ghd10 is orthologous to INDETERMINATE1 (ID1), which promotes flowering in maize (Zea mays) and is identical to the previously cloned genes Rice Indeterminate1 (RID1), Early heading date2 (Ehd2) and Os/d1. Transient expression analysis of the Ghd10-GFP fusion protein in tobacco mesophyll cells showed that this protein is expressed in the nucleus. Ghd10 mRNA accumulated most abundantly in developing leaves and panicle structures, but rarely in roots. Expression analysis revealed that the expression levels of Ehd1, Hd1, RFT1, Hd3a and OsMADS15 decreased dramatically under both short-day and long-day conditions in ghd10.

Conclusion: These results indicate that Ghd10, which encodes a promoter of flowering, influences plant height and panicle development by regulating the expression levels of some flowering-related genes, such as Ehd1, Hd1, OSMADS15 and others. The ghd10 allele is a useful resource for improvement of panicle traits in rice grown in tropical and low-latitude areas.
\end{abstract}

Keywords: Heading date; Plant height; Panicle development; Plant architecture; Map-based cloning

\section{Background}

Plant architecture is one of the most important agronomic traits affecting rice yield formation potential (Wang and Li 2011). As plant height, heading date and panicle development are important components of plant type and yield potential in rice, the study of these factors will pave the way for clarifying the molecular mechanism of

\footnotetext{
* Correspondence: dalizeng@126.com; guolongb@mail.hz.zj.cn

${ }^{\dagger}$ Equal contributors

State Key Laboratory of Rice Biology, China National Rice Research Institute, Chinese Academy of Agricultural Sciences, Hangzhou 310006, China
}

plant morphogenesis and increasing the yield potential of rice using genetic methods (Tripathi et al. 2012; Duan et al. 2013).

Heading date, one of the most important agronomic traits in rice, involves in determining whether currently available varieties of cultivated rice can adapt to specific cropping locations and growing seasons (Izawa 2007; Tsuji et al. 2008). The response to the length of day (LOD) of a rice plant (namely, photoperiod sensitivity [PS]), its temperature sensitivity (TS) and basic vegetative growth (BVG) determine the heading date of the plant. The 
interaction between these factors is thought to determine adaptability of a rice variety (Lee and An 2007). Many genetic studies of heading date have been performed, and several genes controlling heading date in rice have been mapped or cloned (Andrés et al. 2009; Dai and Xue 2010; Hayama et al. 2003; Matsubara et al. 2008; Park et al. 2008; Saito et al. 2011; Wu et al. 2008; $\mathrm{Hu}$ et al. 2012). Yano et al. detected 14 QTLs for heading date (Hd1-Hd14) using several types of progeny derived from a single cross of Nipponbare and Kasalath, subsequently leading to the cloning of Hd1, Hd3a and Hd6 (Yano et al. 2001). Hd1, which is allelic to $\mathrm{Se} 1$, promotes flowering under short-day (SD) conditions while it represses flowering under longday (LD) conditions (Yano et al. 2000). Hd6, involving in rice photoperiod sensitivity, encodes the $\alpha$-subunit of protein kinase $\mathrm{CK} 2 \alpha(\mathrm{CK} 2 \alpha)$ to increase the days-toheading (Takahashi et al. 2001). Another flowering-related gene, $H d 3 a$ acts as a floral activator under SD conditions and is regulated by $H d 1$ (Kojima et al. 2002). The recent studies have shown that $\mathrm{Hd} 3 \mathrm{a}$ can interact with 14-3-3 proteins in the apical cells of shoots to yield a complex that is translocated to the nucleus and binds to the OsFD1 transcription factor to induce the transcription of OsMADS15 (Abe et al. 2005; Taoka et al. 2011). Ehd1 encodes a B-type response regulator that confers short-day promotion of flowering and controls FT-like gene expression independently of $H d 1$ (Doi et al. 2004). Previous studies also found that Ghd7 and Ghd8/DTH8 control multiple-traits of flowering time, plant height and grain numbers per panicle simultaneously (Wei et al. 2010; Xue et al. 2008; Yan et al. 2011).

The transition from vegetative growth to reproductive growth marks the successful completion of floral induction. In addition to this transition, the development of panicle morphology is one of the most important processes concerning rice breeders. Many genes influencing panicle morphology and development have been cloned (Huang et al. 2009; Komatsu et al. 2003; Ikeda et al. 2007; Oikawa and Kyozuka 2009; Qiao et al. 2011; Tabuchi et al. 2011; Terao et al. 2010; Yoshida et al. 2012; Zhu et al. 2010). $D E P 1$ is pleiotropically responsible for dense panicles, high grain number per panicle and erect panicles, and plays an important role in manipulating grain yield (Huang et al. 2009; Yan et al. 2007). Mutation of SP1 results in plants defective in panicle elongation, thus leads to the shortpanicle phenotype (Li et al. 2009). LAX2 (Lax Panicle2) is involved in maintaining axillary meristems (AMs) at the reproductive stage. $L A X 2$ regulates the branching of the aboveground parts of rice throughout plant development, except for the primary branch in the panicle (Komatsu et al. 2001; Komatsu et al. 2003; Oikawa and Kyozuka 2009; Tabuchi et al. 2011). Rice APO1 encoding an F-box protein was isolated from the apo1 mutant exhibiting small inflorescence with a reduced number of branches and spikelets, abnormal floral organ identity and a loss of floral determinacy (Ikeda et al. 2007). Habataki-APO1 rice plants exhibit an increase in the number of primary branches, grain number and yield per plant. APO1 also can enhance the formation of vascular bundle systems, which promotes carbohydrate translocation to panicles and improves lodging resistance in rice (Ookawa et al. 2010). Rice breeders and geneticists have suggested that rice yield formation potential can be increased by further improving rice plant type. IPA1, an ideal plant architecture gene, can coordinate the interaction among all of those yield components (Jiao et al. 2010).

Panicle primordium differentiation and stem elongation are important signals for the transition from vegetative growth to reproductive growth. However, there are few reports about how the vegetative phase (before the transition) is connected to panicle morphology development (after the transition), thereby influencing the yield formation potential of rice. In the current study, we investigated the ghd10 mutant, which exhibits delayed flowering time, tall stalks, increased panicle length and an increased number of primary branches. Map-based cloning revealed that Ghd10 encodes a transcription factor with Cys-2/His2-type zinc finger motifs that is orthologous to INDETERMINATE1 (ID1), which promotes flowering in maize (Zea mays) and was previously identified as Rice Indeterminate1 (RID1), Early Heading Date2 (Ehd2) and OsId1 in rice. Ghd10 acts as a promoter of flowering, mainly through the upregulation of $E h d 1$ and $H d 1$ and the downstream genes $H d 3 a, R F T 1$ and others in the unique genetic network of photoperiodic flowering. How Ghd10 influences plant height, panicle development and even plant architecture has not previously been reported, and the molecular mechanism underlying the activity of Ghd10 remains unclear. The results of this study suggest that Ghd10 plays an important role in regulating yield component traits by increasing plant height and primary branch number in rice, which is dependent on short-day conditions.

\section{Results}

The ghd10 gain-of-function allele in panicle development The ghd10 mutant was obtained from japonica cultivar Wuyunjing 7 (WYJ7) rice plants subjected to EMS treatment. Phenotypic analysis showed that the mutant exhibited delayed flowering in both Fuyang, Zhejiang Province (FY.ZJ) and Lingshui, Hainan Province (LS.HN) compared with the wild-type (Figure 1A). The mutant began to flower on the 149th day after germination under natural SD conditions (LS.HN), with an increase of $54 \mathrm{~d}$ compared with the wild-type. The mutant never flowered during the more than 300-day period after germination under controlled LD conditions, and it also cannot flower under natural LD conditions (from the middle of May to the middle of November, FY.ZJ), whereas the wild-type 

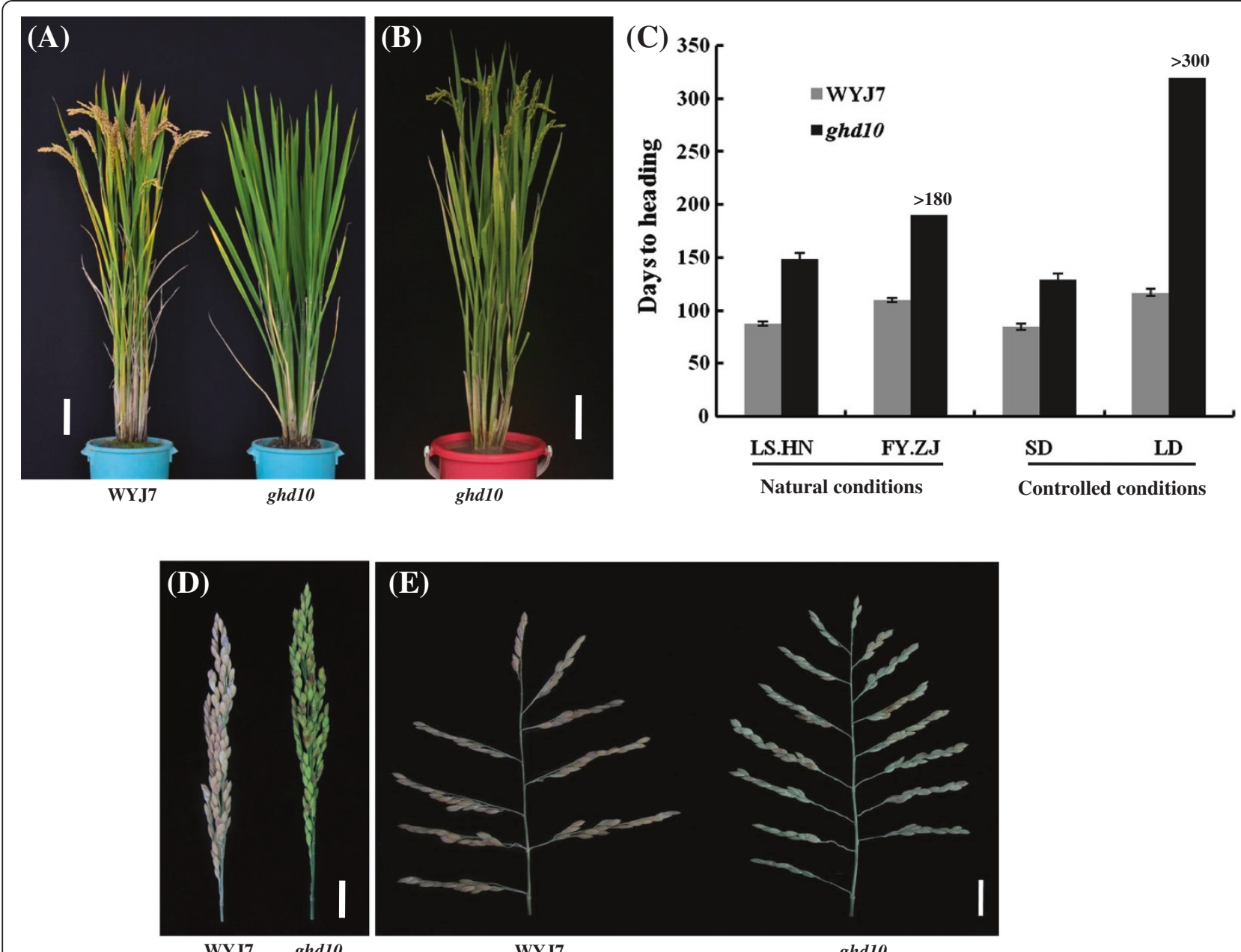

Figure 1 Phenotype comparison of ghd10 mutants and wild-type WYJ7. (A) The phenotype of wild-type WYJ7 (left) and the ghd10 mutant (right) at the mature stage (The plants were cultivated at CNRRI experimental field in Fuyang city, Zhejiang Province, in 2011). (B) The phenotype of the ghd10 mutant at the mature stage (The plants were cultivated at CNRRI experimental field in Lingshui city, Hainan Province, in 2011). (C) Days to heading comparison between the wild-type and the mutant in Fuyang city, Zhejiang Province (FY.ZJ), Lingshui city, Hainan Province (LS. $\mathrm{HN}$ ) and under controlled short-day (SD) and long-day (LD) conditions. (D-E) Increased panicle length (D) and primary branch number (E) in ghd10. Scale bar: (A) and (B), $10 \mathrm{~cm}$; (D) and (E), $2 \mathrm{~cm}$.

plants began to flower on the 110th and 117th day after germination under natural LD conditions (FY.ZJ) and controlled LD conditions, respectively (Figure 1B, C). The difference in flowering time was small in the wild-type plants under SD and LD conditions. Thus, ghd10 exhibited much later flowering time than the wild type under both $\mathrm{SD}$ and LD conditions, and the flowering time delay was more obvious under LD conditions, which indicated that the Ghd10 mutation may have a more severe effect on flowering time under LD conditions than under SD conditions. And these data were consistent with the finding of previous study (Matsubara et al. 2008; Wu et al. 2008).

The prolonged vegetative phase in the mutant caused the panicle length and primary branch number of ghd 10 to increase under natural SD conditions compared with the wild type (Figure 1D, E). Detailed agronomic trait analysis revealed that the panicle length of ghd10 was an average of $17.2 \pm 0.8 \mathrm{~cm}$, which was $8.3 \%$ longer than that of the wild type $(15.4 \pm 0.3 \mathrm{~cm})$, there was a significant difference $(P<0.05)$ between the mutant and wild type. The primary branch number of ghd10 was $14 \pm 0.8$, which was higher than that of wild-type WYJ7 $(9.3 \pm 0.5)$. The main stem of ghd10 was approximately $88.3 \pm 2.7 \mathrm{~cm}$, which was $10.2 \%$ higher than that of the wild type. The primary branch number and plant height were highly significantly different $(\mathrm{P}<0.01)$ between ghd10 and WYJ7. The number of grains per panicle in the mutant was approximately $143 \pm 8.5$, which was $12.4 \%$ more than that of the wild type (124.7 \pm 7.4 on average). These results ran counter to the findings of the previous study (Matsubara et al. 2008; Park et al. 2008; Wu et al. 2008). There was no significant difference in the percentage of seed setting, 1,000-grain weight or 
tiller number between the ghd10 mutant and the wild type (Figure 2A-H). These results indicate that the Ghd10 mutation increases the plant height, panicle length and primary branch number in addition to delaying the heading date.

\section{Late flowering increases the leaf number per tiller in ghd10 and is independent of the leaf emergence rate}

Since the leaf emergence rate reflects the growth and developmental stage or growth rate of rice, we identified the specific growth and developmental periods of the plants by examining the leaf number on the main stem. To determine whether the reduced growth rate affected late flowering in $g h d 10$, we compared the leaf emergence rate between ghd10 and the wild type under natural SD (NSD) and natural LD (NLD) conditions up to the 160th day after germination. Before flowering, the leaf emergence rate of ghd10 was almost the same as that of the wild type under both NSD and NLD conditions. Under NSD conditions, ghd10 flowered after the 17th leaf emerged, which occurred by the 149th day after germination, and the mutant developed 21 leaves by the 160th day after germination (Figure 3A). The prolonged heading date increased the number of leaves per tiller in addition to increasing the panicle length and primary branch number (Figure 1C, D), and the nodes were wrapped by the leaf sheath of the additional leaves in ghd10 under NSD conditions, unlike in WYJ7 (Figure 3B-E). Thus, the mutation of Ghd10 did not affect the growth rate of the mutant, but the mutation produced a gain-of-function in inflorescence development. These results reveal that Ghd10 controls the floral transition and the subsequent development of inflorescences in rice.

\section{The point mutation of Ghd10 is responsible for the ghd10 phenotype}

To determine whether the ghd10 phenotype is controlled by a single gene, we performed genetic analysis of reciprocal crosses between ghd10 and the japonica cultivars NIP, WYJ7 and CJ06. The results showed that ghd10 was a recessive mutant, based on examination of individuals of the $\mathrm{F}_{2}$ segregating population. The ratio of individuals with normal flowering to late flowering was $3: 1\left(x^{2}<x^{20.05,1}=3.84\right)$, indicating that the ghd10 phenotype was controlled by a single recessive gene (Additional file 1: Table S2).

Bulked Segregant Analysis (BSA) was used to produce a primary map of Ghd10. PCR genotyping was carried out using a bulk DNA pool from 36 ghd10/NJ06 $\mathrm{F}_{2}$ individuals with the mutant phenotype, and 136 SSR and STS markers scattered on all of the rice chromosomes were used to determine the approximate map position of Ghd10. Ghd10 was primarily located on chromosome 10 , closely linked to RM5689, at a genetic distance of $4.2 \mathrm{cM}$ (Figure 4A). To further fine map Ghd10, we designed new STS and InDel markers next to RM5689 based on the sequence difference between japonica rice variety Nipponbare and indica

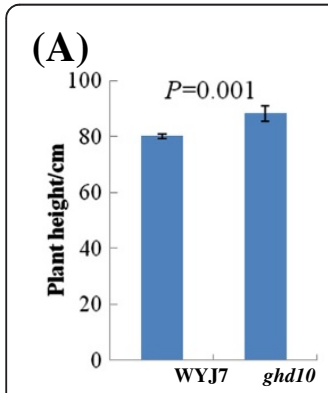

(F)

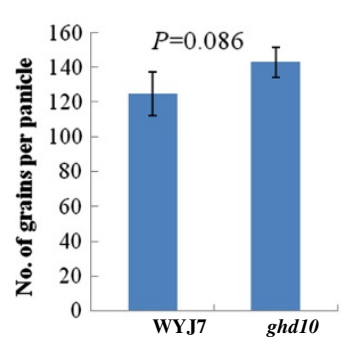

(B)

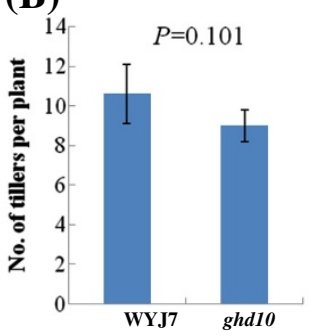

(G)

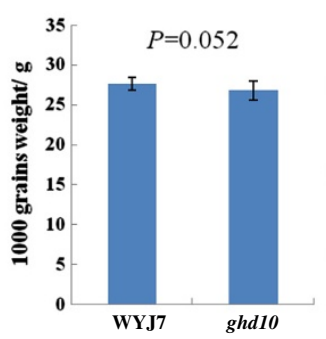

(C)

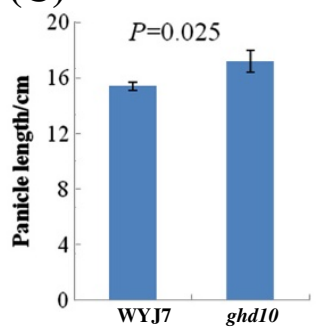

(H)

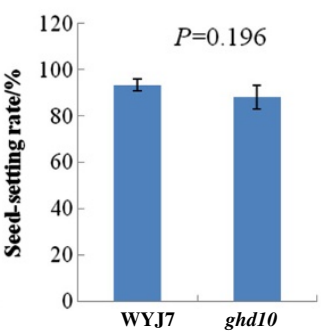

(D)

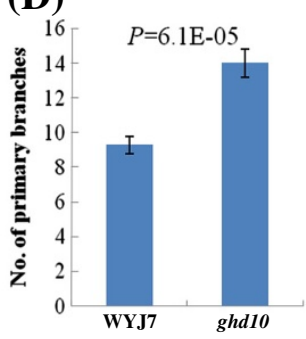

(I)

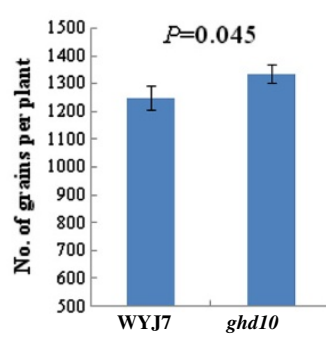

(E)

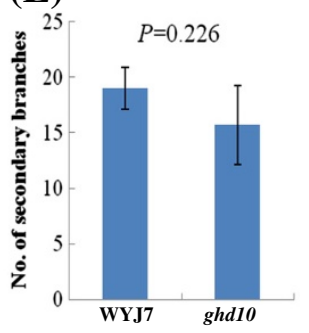

(J)

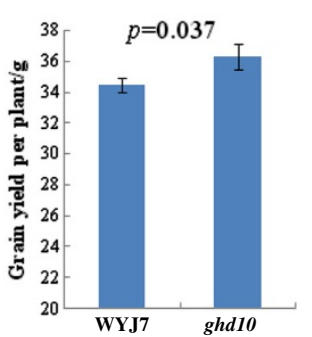

Figure 2 Agronomic trait performance of ghd10 and WYJ7 plants. (A) Plant height. (B) Number of tillers. (C) Panicle length. (D) Number of primary branch per panicle. (E) Number of secondary branch per panicle. (F) Number of grains per panicle. (G) 1,000-grain weight. (H) Seed-setting rate. (I) Number of grains per plant. (J) Grain yield per plant. WYJ7 and ghd10 plants were grown in a standard paddy field with a distance of $16^{*} 20 \mathrm{~cm}$ under conventional cultivation conditions in Lingshui city, Hainan Province, in 2012. All data are shown as mean \pm s.e.m. A Student's t-test was used to generate the $P$ values. 


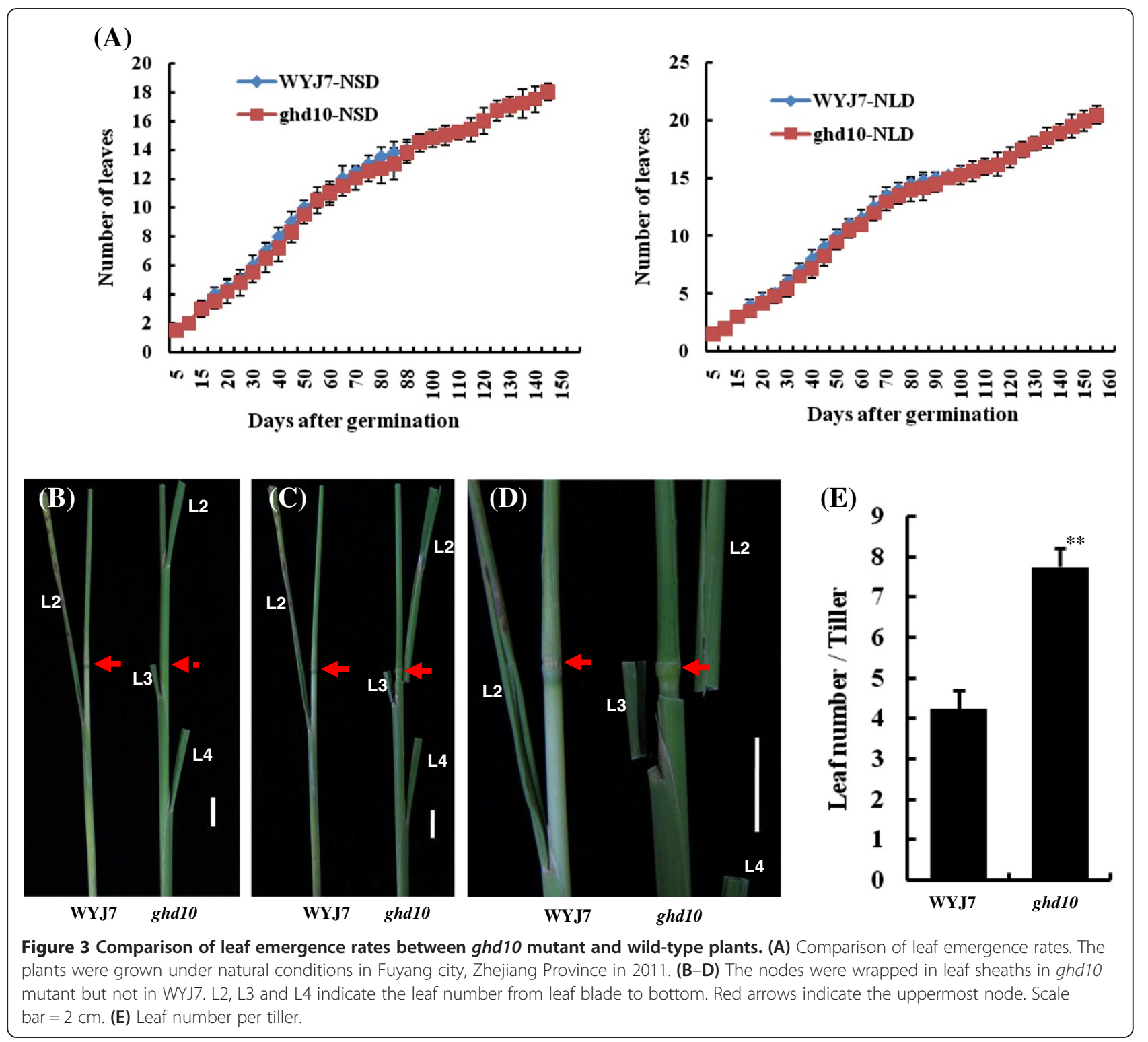

variety 9311 (http://www.gramene.org/resources/). The polymorphism primers were subsequently used to screen 1,611 individual genotypes, which mapped the Ghd10 locus between two STS markers, k10-5 and k10-3, within a 29-kb physical interval (Figure 4B). Finally, we obtained one predicted ORF, Os10g0419200, according to the genomic annotation database (RAP-DB, http://rapdb.dna. affrc.go.jp/). Sequence analysis revealed only one single nucleotide polymorphism (SNP) in the Os10g0419200 coding sequence. This mutation resulted in an amino-acid substitution from proline (Pro) to leucine (Leu) in the 158th residue (Figure 4C). Genetic complementation verified the identity of the Ghd10 candidate gene. We introduced the plasmid pGhd10, which contained the entire Os10g0419200 ORF, and pCK empty vector as a negative control, into ghd10 (Figure 4D). The phenotype of ghd10 was restored to normal in the three transgenic pGhd10 lines, whereas all six of the pCK lines of failed to recover the wild type phenotype, which demonstrated that the cloned candidate gene indeed represented Ghd10 (Figure 4F, G). In summary, we cloned Ghd10 and determined that a point mutation was responsible for the phenotype of ghd10.

Ghd10 encodes a putative transcription factor with zinc finger motifs localized in the nucleus

Bioinformatic analysis indicated that the coding sequence (CDS) of Ghd10 consisted of 1,428 nucleotides encoding a 475 amino-acid protein (Figure 4E). The deduced amino acid sequence of Ghd10 contained two C2H2-type and two C2HC-type zinc finger motifs, previously designated as the ID domain (Kozaki et al. 2004), implying that Ghd10 

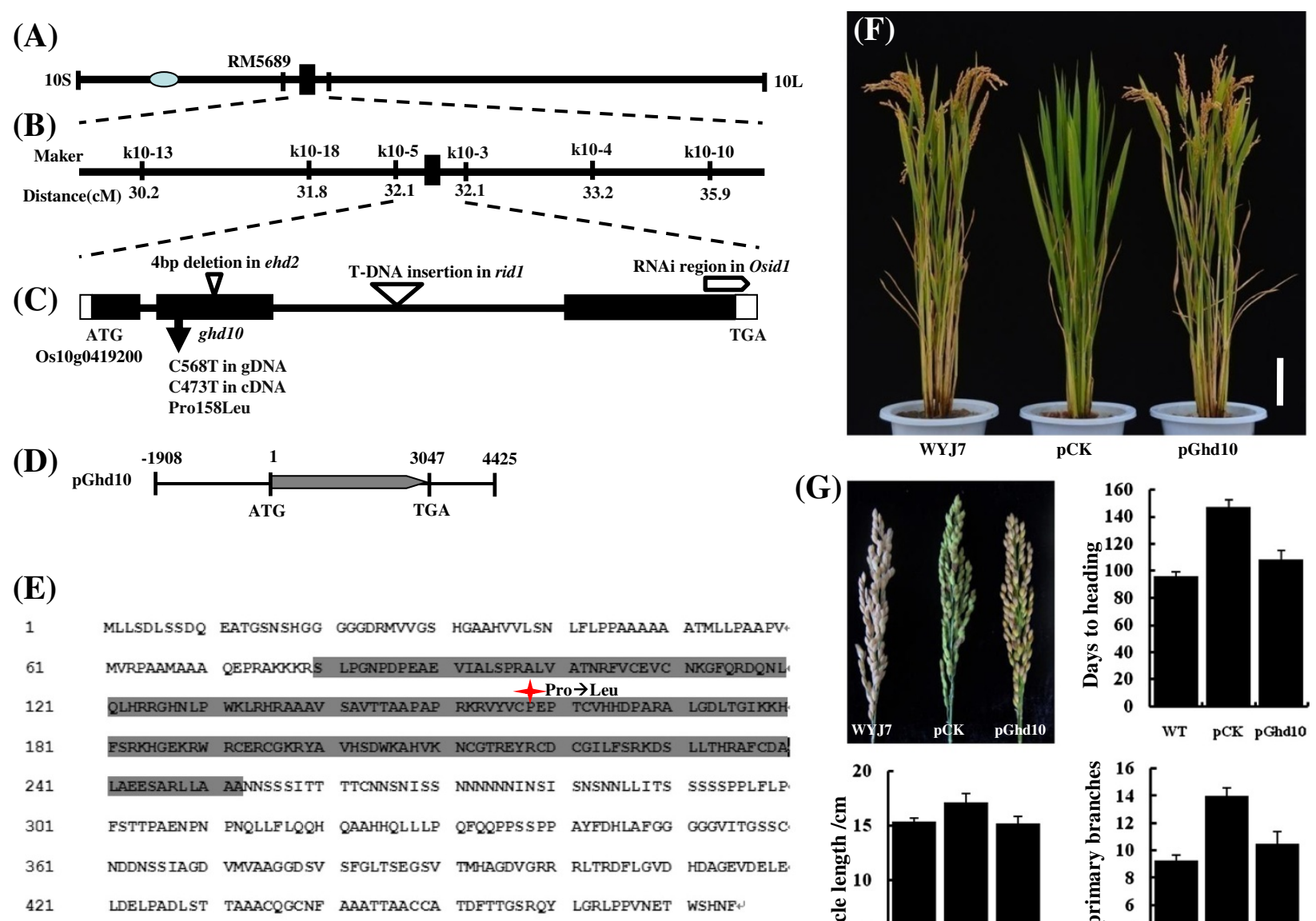

(G)
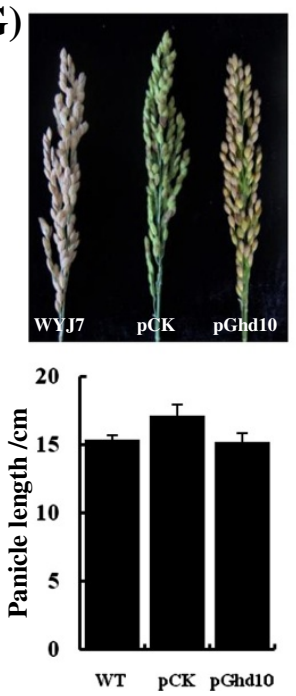
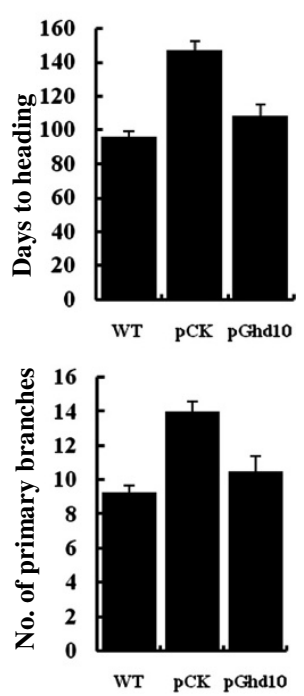

Figure 4 Map-based cloning of Ghd10. (A) Location of Ghd10 on the long arm of rice chromosome 10. (B) The gene was mapped to the interval between molecular markers k10-5 and k10-3 on Nipponbare BAC AC027658 on the basis of genotyping 1,647 $\mathrm{F}_{2}$ progeny. (C) Allelic variation of the Ghd10 sequence. (D) Complementation plasmid. The plasmid pGhd10 contained the entire Ghd10 gene sequence. (E) Gray box indicates the putative ID domain containing two $\mathrm{C} 2 \mathrm{H} 2$ and two $\mathrm{C} 2 \mathrm{HC}$ zinc fingers. Red asterisk indicates the amino acid substitution in the mutant. (F) Phenotypes of transgenic lines. PCK indicates that the empty vector was used in the transgenic line as the negative control. Scale bar $=10 \mathrm{~cm}$. (G) Panicle morphology comparison and panicle related-traits analysis among the wild type, negative control and transgenic line. $S c a l e ~ b a r=2 \mathrm{~cm}$.

encoded a zinc finger transcription factor. To assess the subcellular localization of Ghd10, we performed a transient expression experiment to analyze Ghd10 expression in tobacco epidermal cells. The full-length cDNA was amplified using the following primer pair: CDS-F (5'-ATGTTGCT GTCTGATCTCTCGTCTGA-3'), CDS-R (5' -GAAGTTG TGGCTCCACGTCTCGTTCA-3'). The $C$ terminal of Ghd10 was fused to GFP under the control of the cauliflower mosaic virus (CaMV) $35 \mathrm{~S}$ promoter, which was injected into the leaves of one-month-old tobacco seedlings. The transformed tobacco plants were incubated for approximately $48 \mathrm{~h}$ at $24^{\circ} \mathrm{C}$ under a $14 \mathrm{~h} \mathrm{light} / 10 \mathrm{~h}$ dark cycle. Small pieces of injected leaves were then examined under a confocal fluorescence microscope. The results showed that the Ghd10-GFP fusion protein localized exclusively in the nucleus, in contrast to the control, in which GFP signals were observed throughout the tobacco cells (Figure 5A, B). The results of Ghd10-GFP subcellular localization in tobacco mesophyll cells were consistent with the previous study of RID1-GFP in onion epidermal cells (Wu et al. 2008) and OsID1-GFP in roots of transgenic plants (Park et al. 2008).

\section{Expression pattern of Ghd 10}

Previous studies of the maize ZmID1 gene revealed that mRNA transcripts of the gene were primarily restricted to young leaves that were folded inside of maize stems (Colasanti et al. 1998; Wong and Colasanti 2007; Matsubara et al. 2008; Park et al. 2008; Wu et al. 2008). Therefore, we first examined the expression levels of Ghd10 transcripts of young plants at the vegetative stage (Figure 5C) using quantitative real time-PCR under NLD 


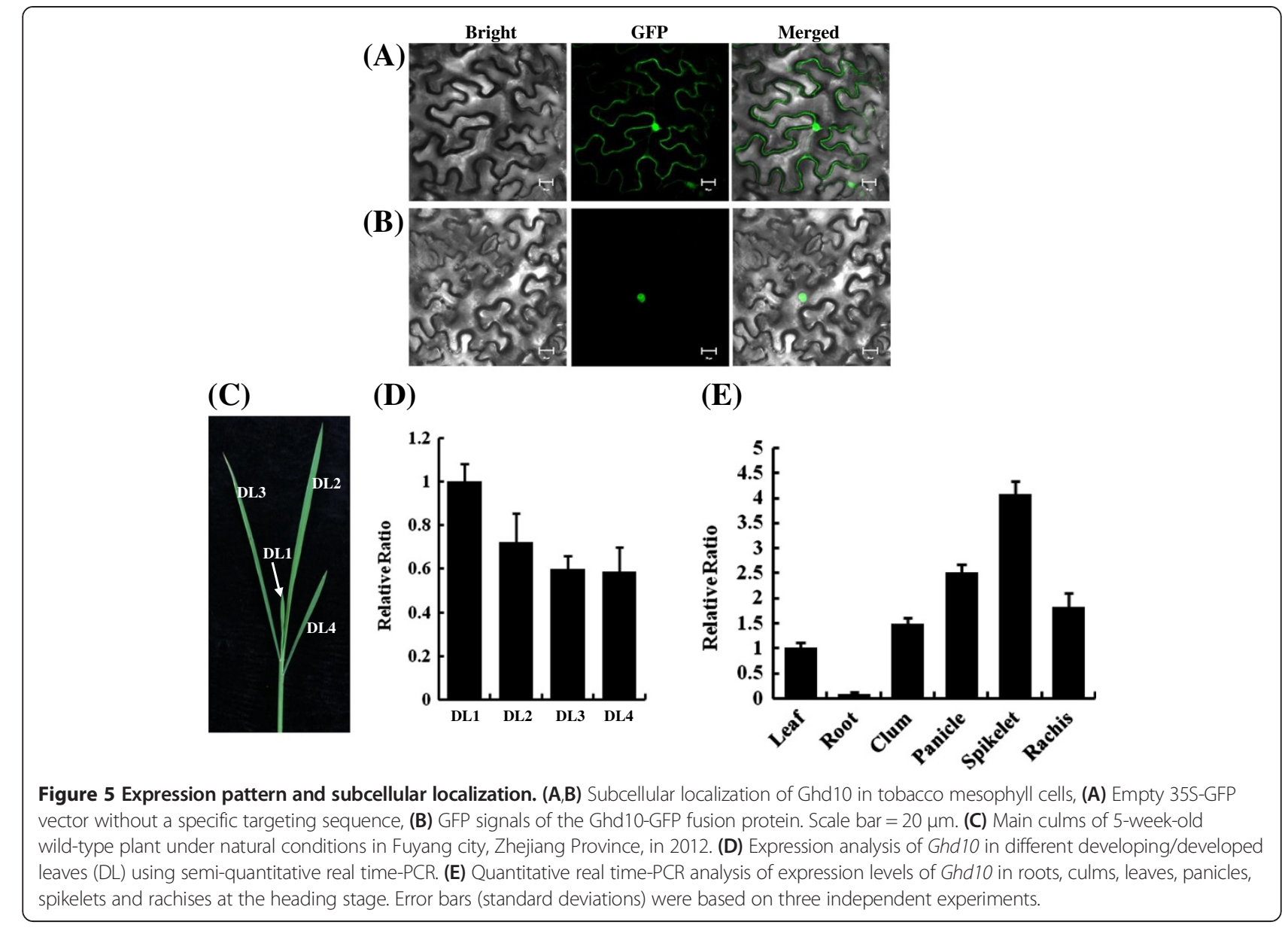

conditions. Most of the Ghd10 mRNA accumulated at high levels in developing leaves (DL1) within the leaf sheath. The expression level of Ghd10 was higher in DL1 than in older leaves (DL2, DL3 and DL4), but there was no significant difference in expression levels between DL3 and DL4 (Figure 5D). These results indicated that during leaf development, the expression of Ghd10 in leaves gradually declined and was then maintained at a stable level. We also examined the expression patterns of Ghd10 in WYJ7 roots, culms, leaf blades, panicles, spikelets and rachises at the heading stage by RT-PCR. Ghd10 was expressed in all of the tissues examined, with relatively high levels of expression in the spikelets, panicles and rachises and lower expression levels in the culms and mature leaf blades, but the transcripts were difficult to detect in the roots (Figure 5E). The observation that Ghd10 expression varied in an organ-specific manner was consistent with the varied phenotypes observed in ghd10. These results suggest that Ghd10 first accumulates in developing leaves and interacts with an endogenous or exogenous signal to control the induction of the transition from vegetative to reproductive development in the early vegetative growth stage, and this gene is highly expressed in panicles to regulate panicle development after the transition to the reproductive growth stage occurs.

\section{Ghd10 regulates the expression levels of floral pathway genes}

To examine the accumulation of mRNAs of Ghd10, Ehd1, $H d 1, H d 3 a, R F T 1$ and other flowering-related genes during development, we harvested developed leaves from wildtype and ghd10 plants at the heading stage and analyzed the leaves by quantitative RT-PCR. The samples were collected $0.5 \mathrm{~h}$ after dawn under SD and LD conditions (Figure 6A). There was no significant difference in the levels of Ghd10 mRNA accumulation between ghd10 and wild-type plants under SD or LD conditions. These results suggest that the point mutation in Ghd10 may not affect the transcription level of Ghd10, suggesting that the resulting change in a single amino acid residue in Ghd10 may affect the advanced structure and function of this protein. By contrast, the expression levels of Ehd1 and $H d 1$ dramatically decreased under both SD and LD conditions. A previous study demonstrated that the combination of mutant alleles of $H d 1$ and Ehd1 in T65 rice can reduce the number of primary branches in the panicle, resulting in 


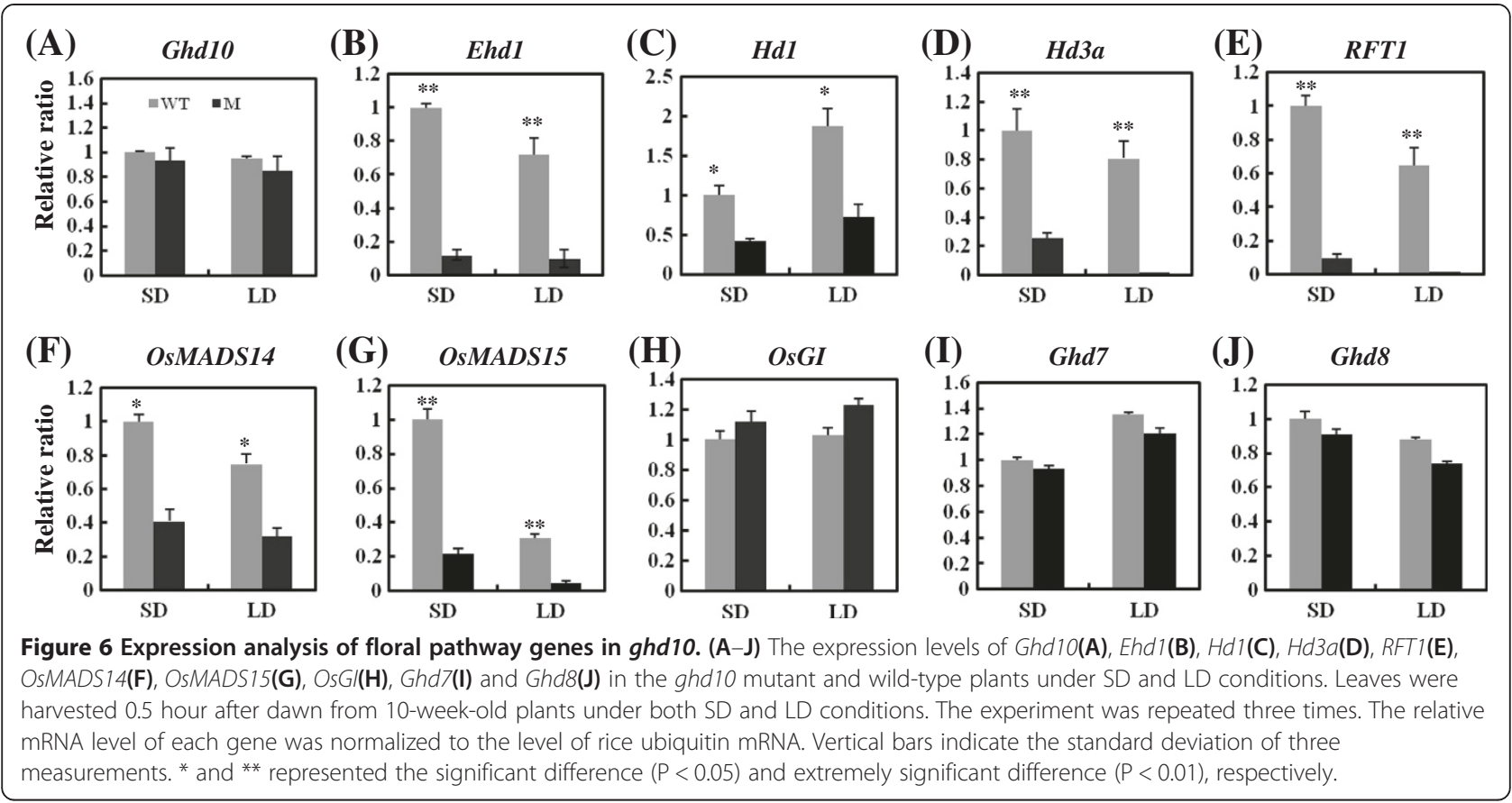

smaller spikelet numbers per panicle (Endo-Higashi and Izawa 2011), which suggested that the combination of $h d 1$ and $e h d 1$ alleles, or the downregulated expression of $H d 1$ and Ehd1, may increase the number of primary branches and the number of spikelets per panicle. To help confirm this hypothesis, we examined changes in the expression levels of $H d 1$ and $E h d 1$ in ghd10 versus wild-type plants (Figure 6B, C). And the result of expression pattern of $H d 1$ and Ehd1 supported our speculation and previous reports (Endo-Higashi and Izawa 2011). Hd3a, RFT1, OsMADS14 and OsMADS15 encode key regulators in the floral pathway and function downstream of $H d 1$ and Ehd1. The expression patterns of these genes matched that of $H d 1$ and Ehd1 (Figure 6D-G). The expression of OsGI slightly increased under both SD and LD conditions in ghd10, but the level of increase was not significant (Figure 6H). The expression levels of Ghd7 and Ghd8 were not significantly difference between WYJ7 and gdh10 under both growth conditions (Figure 6I, J). While the expression levels of panicle development-related genes, such as DEP1, FZP, $L A X 1$ and $S P 1$, were not significantly different between WYJ7 and $g d h 10$ under both growth conditions (Additional file 1: Figure S1). These results suggest that Ghd10 regulates flowering time, panicle development and yield formation may independent on Ghd7 and Ghd8.

\section{Discussion}

Ghd10 controls yield-related traits in addition to flowering time

Recent studies have shown that flowering-related genes in rice, Arabidopsis and other plants play important roles in various physiological processes other than flowering. In rice, increased tillering occurs in transgenic rice plants expressing the gene encoding florigen Hd3a-GFP fusion protein under the control of phloem-specific promoters, in addition to accelerated flowering (Tamaki et al. 2007). Nano scale proteomics revealed the presence of regulatory proteins including three FT-like proteins, OsFTL12, OsRCN3 and OsMFT1, in rice phloem sap (Aki et al. 2008). Moreover, growth analysis of lateral shoots in mutants of FLOWERING LOCUS T (FT) and TWIN SISTER OF FT (TSF) in Arabidopsis reveals a delay in the onset of outgrowth and a reduction of the growth rate in $f t$ plants under LD conditions and in tsf plants under SD conditions. In addition, two florigen genes, $F T$ and TSF, play important roles in linking the floral transition and lateral shoot development to maximize the reproductive success of Arabidopsis plants. The promotion of lateral shoot development by these two florigen genes is independent of their effect on the floral transition of the primary shoot (Hiraoka et al. 2013). All of these studies suggest that flowering time and other traits can be affected by a signal gene in plants. The interaction between the two florigen genes may also influence yield-related traits in rice. As previously reported, $H d 1$ promotes flowering under SD conditions while inhibiting flowering under LD conditions (Yano, et al. 2000). Ehd1 promotes the floral transition preferentially under SD conditions, even in the absence of functional alleles of $H d 1$ (Doi et al. 2004). In a study by Endo-Higashi and Izawa, four rice lines with different flowering-time genotypes (hd1 ehd1, hd1 Ehd1, Hd1 ehd1 and Hd1 Ehd1) were 
grown under distinct photoperiod conditions. The authors compared the effects of flowering-time genes on traits related to plant architecture using genotype-treatment combinations that resulted in similar flowering times in the lines that were examined. The results showed that the combination of $H d 1$ and Ehd 1 reduces the primary branch number in a panicle, resulting in a reduced spikelet number per panicle (Endo-Higashi and Izawa 2011), which demonstrates that the interaction between $H d 1$ and Ehd1 influences primary branch development independent of controlling flowering time. In the current study, the ghd 10 plants displayed outstanding yield traits, including increased panicle length, primary branch number and plant height, in addition to late heading date under SD conditions. The expression levels of two florigen genes (Hd3a and RFT1), two rice AP1 genes (OsMADS14 and OsMADS15) and the combination of Ehd1 and $H d 1$ dramatically declined in ghd10 plants under both SD and LD conditions. Combining the results of expression analysis and phenotypic variation analysis in $g h d 10$ plants, we conclude that the ghd10 allele increases primary branch number, panicle length and plant height, and this allele also controls flowering time in response to SD growth conditions.

\section{The long vegetative growth period increases yield formation potential}

Flowering time, one of the most important agronomic traits in rice, not only determines or influences the growth period of rice, but it also affects other agronomic traits (Hori et al. 2012). In studies of crop productivity, flowering time control was often associated with yield-related traits, as a prolonged vegetative period results in greater biomass accumulation, which can be conductive to the accumulation of more photosynthetic products. In fact, Ghd7 has been shown to affect grain number in field tests (Xue et al. 2008). Ghd7, which was isolated from natural rice variants, encodes a CCT-domain protein and has major effects on heading date, plant height and the number of grains per panicle. Under LD conditions, the expression of Ghd7 is enhanced, leading to delayed flowering time and increased plant height and panicle size. In a study comparing 19 genotypes of rice from rice growing areas representing a wide range of geographic regions in Asia, rice varieties with early heading, reduced plant height and panicle size were shown to have a deletion of the Ghd7 locus. When Ghd 7 was transferred to plants, the transformants exhibited delayed flowering, increased plant height and panicle size (Xue et al. 2008).

Rice Ghd8/DTH8, a major QTL with pleiotropic effects on grain yield, heading date and plant height, encodes the OsHAP3 subunit of a CCAAT-box binding protein (HAP complex). Ghd8/DTH8 is sensitive to photoperiod, and a mutation in this gene makes a plant insensitive to photoperiod and reduces plant height. Under LD conditions, plants with a deletion of Ghd8/DTH8 exhibit delayed heading date and an increased plant height and number of grains per panicle when a functional Ghd8/DTH8 allele is transferred to the plant (Wei et al. 2010; Yan et al. 2011). Here, we showed the Ghd10 mutation has pleiotropic effects on grain yield, heading date and plant height and functions like Ghd7 and Ghd8/DTH8. A longer vegetative growth period allows more photosynthate (the source) to be transferred to grains, i.e., the sink capacity increases, after flowering. When a plant has a large sink capacity and the flow between the sink and the source is unimpeded, the yield formation potential increases.

\section{The allele ghd 10 improves panicle traits in rice}

Previous studies have revealed that the functions of rice Ehd2/RID1/OsID1 and maize ID1 are roughly conserved with respect to their role in flowering promotion and the tissue-specific expression of these genes, as there is high identity (82\%) between their zinc finger domains (Matsubara et al. 2008; Park et al. 2008; Wu et al. 2008; Colasanti et al. 1998; Wong and Colasanti 2007). However, there are some functional differences between these genes. For instance, severe $i d 1$ mutants in maize exhibit an abnormal transition to floral development and produce aberrant inflorescences with vegetative shoot characteristics (Colasanti et al. 1998). Such a morphological aberration has not been observed in ehd2, rid1 or Osid1 plants (Matsubara et al. 2008; Park et al. 2008; Wu et al. 2008). Therefore, species specificity of the genetic control of floral development may result in differences in the phenotypes caused by the mutation of orthologous gene, such as rice Ehd2/RID1/OsID1 and maize ID1.

Phenotypic differences caused by the mutation of allelic genes also exist between subspecies or varieties of rice (Matsubara et al. 2008; Park et al. 2008; Wu et al. 2008). In this study, Ghd10 was cloned using a map-based approach, and the locus was shown to encode a zinc finger transcript factor by transgenic complementation studies. Furthermore, the Ghd10 locus of the ghd10 mutant was shown to be allelic to Ehd2/RID1/OsID1. However, there were differences between the phenotypes of ghd10 that we observed and the phenotypes reported for ehd2, rid 1 and osid1. In rice ehd 2 plants, a 4-bp insertion within the second exon of the putative zinc finger protein encoded by the Ehd2 allele resulted in a premature stop codon in the open reading frame of this gene. The change in the coding sequence gave rise to plants with late flowering and smaller panicles compared with the wild type (Matsubara et al. 2008). In another study, rid1 plants exhibited "eternal" vegetative growth and a neverflowering phenotype under both SD and LD conditions, which was caused by a T-DNA insertion in the second intron of the gene encoding a putative zinc finger protein 
(Wu et al. 2008). However, in the current study, the point mutation in the zinc finger motif of the Ehd2/RID1/OsID1 protein in the ghd10 plants conferred obvious advantages to yield formation traits, including increased plant height, panicle length, primary branch number and number of spikelets per panicle, compared with the wild type and three other allelic mutants. This difference may have resulted from differences in the position/interval of the mutation in the coding sequences or differences in the genetic background of the allelic mutants examined; this topic remains to be explored in the future. We also plan to excavate as many ghd10 alleles as possible and screen the more favorable alleles for rice production practice using a functional genomics approach.

Overall, the cloning of Ghd10 has provided a rare opportunity for studying the molecular mechanisms underlying the association between yield-related traits and flowering. The ghd10 allele is a useful resource for rice breeding to improve panicle traits in rice grown in tropical and low-latitude areas.

\section{Conclusion}

The ghd10 mutant is characterized by delayed flowering time, tall stalks, increased panicle length and primary branch number and other phenotypes. Map-based cloning revealed that Ghd10 encodes a transcription factor with Cys-2/His-2-type zinc finger motifs orthologous to the INDETERMINATE1 (ID1) gene in maize. Expression analysis indicated that Ghd10 acts as a flowering promoter and influences plant height and panicle development by regulating the expression levels of some flowering-related genes, such as Ehd1, Hd1, OsMADS15 and others. The ghd10 allele represents a useful resource for improving yield-related traits in rice bred for tropical and low-latitude areas.

\section{Materials and methods}

\section{Plant materials and growth conditions}

The ghd10 mutant was derived from an $\mathrm{M}_{2}$ population of the japonica rice cultivar Wuyunjing 7 (WYJ7) after EMS mutagenesis (After soaking for 24 hours, transfer the seeds into $1.5 \%(\mathrm{v} / \mathrm{v})$ EMS solution at 28 degree environmental temperature and treat with 12 hours, then washing the seeds 10 hours in flowing water and accelerating germinations. See the Ref. of Guo et al. (2006)). The ghd10 mutant and progeny all exhibited delayed flowering time, increased primary branch number and tall stalks, inherited. The japonica cultivars Nipponbare (NIP), Wuyunjing 7 (WYJ7) and Chunjiang 06 (CJ06) and the indica cultivar Nanjing 06 (NJ06) were used for segregating population construction. All plants were grown in a paddy field at the China National Rice Research Institute (CNRRI), Fuyang, Zhejiang Province, China and Lingshui, Hainan Province, China.

\section{Photoperiod sensitivity test}

To determine whether the mutation of ghd10 was associated with photoperiod, 25-day-old ghd10 and WYJ7 rice seedlings were cultivated in growth chambers (SANYO, Versatile Environmental Test Chamber, MLR-351H) under both continuous SD conditions (9.5 h light/14.5 h dark) and LD conditions (14.5 h light/ $9.5 \mathrm{~h}$ dark) with diurnal variation of temperature and relative humidity (Additional file 1: Table S1). The days to heading of ghd10 and WYJ7 under both growth conditions were recorded.

\section{Genetic analysis and map-based cloning}

For genetic analysis to determine whether a dominant or recessive, single or multiple gene controls the ghd10 phenotype, reciprocal crosses between ghd10 and the japonica cultivars NIP, WYJ7 and CJ06 were conducted. The $\mathrm{F}_{2}$ segregation populations were used for a $\chi^{2}$ test.

For map-based cloning, an $\mathrm{F}_{2}$ segregation population for mapping derived from a cross between the ghd10 mutant and the indica cultivar NJ06 was constructed to identify the gene in the mutants. The parents and 6,846 $\mathrm{F}_{2}$ individuals were planted in a paddy field, among which 978 with the mutant phenotype were used to map the Ghd10. PCR genotyping was carried out using a DNA bulk-pool from 36 ghd10/NJ06 $\mathrm{F}_{2}$ individuals with the mutant phenotype, and a total of 136 SSR and STS markers scattered among all of the rice chromosomes were used to determine the approximate map position of the ghd10 locus on the rice chromosomes according to Temnykh et al. (2000) and McCouch et al. (2002).

\section{DNA extraction and molecular marker analysis}

Total genomic DNA was extracted from fresh leaves using the cetyltrimethylammonium bromide (CTAB) method with minor modifications. For mapping, SSR markers were obtained from the Gramene database (www. gramene.org). PCR-based STS and InDel markers were developed based on the sequence differences between japonica rice variety Nipponbare and indica variety 9311 (http://www.gramene.org/resources/). The primer sequences of the molecular markers used are listed in Additional file 1: Table S3. Primers flanking the InDel and SNP polymorphisms were designed using the Primer Premier 5.0 program and tested on the parent varieties by agarose gel electrophoresis.

\section{Complementation test}

A 6,334-bp genomic DNA fragment containing the entire Ghd10 coding region and upstream and downstream sequences of Ghd10 was subcloned from the BAC clone AC027658 and inserted into the binary vector pCAMBIA1300 to generate the transformation vector pGhd10 for use in the complementation test. The pGhd10 and pCAMBIA1300 (pCK) plasmids were introduced into 
ghd10 by Agrobacterium-mediated transformation using Agrobacterium tumefaciens strain EHA105.

\section{Subcellular localization of Ghd10}

To investigate the subcellular localization of Ghd10, the Ghd10 ORF without a termination codon was cloned into the pCaMV35S-GFP binary vector, placing Ghd10 upstream of the GFP coding sequence to create an in-frame fusion of Ghd10 cDNA and the GFP reporter gene. The fusion constructs, as well as the control, were transformed into tobacco (Nicotiana benthamiana) epidermal leaf cells by Agrobacterium-mediated injection. The transformed tobacco epidermal cells were incubated for approximately $48 \mathrm{~h}$ at $24^{\circ} \mathrm{C}$ under $10 \mathrm{~h}$ dark $/ 14 \mathrm{~h}$ light conditions. The cells were then examined under a confocal fluorescence microscope (Carl Zeiss, LSM 780).

\section{RNA extraction and quantitative real-time PCR analysis}

Total RNA was extracted from the leaves of plants that were grown under SD or LD conditions using a Total RNA Extraction Kit (Axygen, cat No,AP-MN-MS-RNA-250). Total RNA was treated with RNase-free DNase (Promega; http://www.promega.com) and used for complementary DNA synthesis using a ReverTra Ace qPCR-RT Kit (TOYOBA, Japan) as described by the manufacturer. Real-time PCR was performed using $2 \times$ SYBR Green PCR Master Mix (Applied Biosystems) in an Applied Biosystems 7900HT Real-Time PCR System with at least three PCR replicates per sample. The PCR conditions were $2 \mathrm{~min}$ at $50^{\circ} \mathrm{C}$, then $10 \mathrm{~min}$ at $95^{\circ} \mathrm{C}$, followed by 40 cycles of $15 \mathrm{~s}$ at $95^{\circ} \mathrm{C}$ and $1 \mathrm{~min}$ at $60^{\circ} \mathrm{C}$. The relative expression level of each transcript was compared with that of $R U B Q 2$. The specific primers used for quantification of Hd3a, RFT1, OsMADS15, OsMADS14, Hd1, Ehd1, Ghd7, Ghd8, DEP1, FZP, LAX1, SP1 and RUBQ2 mRNA expression are listed in Additional file 1: Table S4.

To analyze the expression pattern of Ghd10, total RNA was extracted from roots, culms, leaves, leaf sheaths, panicles, rachises and spikelets of plants at the heading stage and from leaves at various developmental stages from plants at the tillering stage using a Total RNA Extraction Kit (Axygen, cat No,AP-MN-MS-RNA-250). The specific primers 5' -CGACAATAGCTCGATCGCC-3' and 5' -AA GCCCGAAGCTGACACTGT-3' were used to quantify the expression of Ghd10 mRNA. The relative expression levels in different tissues were determined to compare the expression of Ghd10 with that of RUBQ2.

\section{Additional file}

Additional file 1: Table S1. The diurnal variation of light and temperature in the illumination incubator. Table S2: Genetic analysis of ghd10. Table S3: Molecular markers used for Ghd10 mapping and sequencing. Table S4: Real-time PCR primers used in this study. Figure S1:
Transcriptional levels in ghd10 and WYJ7 of (A) DEP1, (B) FZP, (C) LAX1, (D) SP1. Data are displayed as the ratio of expression to rice $R U B Q 2$ RNA, data given as mean \pm standard error. All assays were repeated at least three times.

\section{Competing interests}

The authors declared that they have no competing interests.

\section{Authors' contributions}

SH performed the mapping, cloning and analyzing the Ghd10 gene, and wrote the manuscript. GD performed the characterization of ghd10. JX and YS performed the rice transformation. ZS, WY, GL, YL, BZ and JH investigated agronomic traits. QQ conceived the proposal, generated financial support for the study and corrected the final manuscript. DZ and LG generated financial support for the study and corrected the final manuscript. All authors read and approved the final manuscript.

\section{Acknowledgements}

This research was supported by grants from the National Science Foundation of China (31271700), National Basic Research Program of China (2013CBA01405) and the Ministry of Agriculture of China (2013ZX08001-003).

Received: 23 May 2013 Accepted: 11 October 2013

Published: 18 October 2013

\section{References}

Abe M, Kobayashi Y, Yamamoto S, Daimon Y, Yamaguchi A, Ikeda Y, Ichinoki H, Notaguchi M, Goto K, Araki T (2005) FD, a bZIP protein mediating signals from the floral pathway integrator FT at the shoot apex. Science 309:1052-1056

Aki T, Shigyo M, Nakano R, Yoneyama T, Yanagisawa S (2008) Nano scale proteomics revealed the presence of regulatory proteins including three FT-like proteins in Phloem and Xylem saps from rice. Plant Cell Physiol 49:767-790

Andrés F, Galbraith DW, Talón M, Domingo C (2009) Analysis of PHOTOPERIOD SENSITIVITY5 sheds light on the role of phytochromes in photoperiodic flowering in rice. Plant Physiol 151(2):681-690

Colasanti J, Yuan Z, Sundaresan V (1998) The indeterminate gene encodes a zinc finger protein and regulates a leaf-generated signal required for the transition to flowering in maize. Cell 93:593-603

Dai C, Xue HW (2010) Rice early flowering1, a CKl, phosphorylates DELLA protein SLR1 to negatively regulate gibberellin signalling. EMBO J 29(11):1916-1927

Doi K, Izawa T, Fuse T, Yamanouchi U, Kubo T, Shimatani Z, Yano M, Yoshimura A (2004) Ehd1, a B-type response regulator in rice, confers short-day promotion of flowering and controls FT-like gene expression independently of $\mathrm{Hd} 1$. Genes Dev 18(8):926-936

Duan MJ, Sun ZZ, Shu LP, Tan YN, Yu D, Sun XW, Liu RF, Li YJ, Gong SY, Yuan DY (2013) Genetic analysis of an elite super-hybrid rice parent using high-density SNP markers. Rice 6:21

Endo-Higashi N, Izawa T (2011) Flowering time genes Heading date 1 and Early heading date 1 together control panicle development in rice. Plant Cell Physiol 52(6):1083-1094

Guo LB, Chu CC, Qian Q (2006) Rice mutants and functional genomics. Chin Bull Bot 23:1-13

Hayama R, Yokoi S, Tamaki S, Yano M, Shimamoto K (2003) Adaptation of photoperiodic control pathways produces short-day flowering in rice. Nature 422(6933):719-722

Hiraoka K, Yamaguchi A, Abe M, Araki T (2013) The florigen genes FT and TSF modulate lateral shoot outgrowth in Arabidopsis thaliana. Plant Cell Physiol 54(3):352-368

Hori K, Kataoka T, Miura K, Yamaguchi M, Saka N, Nakahara T, Sunohara Y, Ebana K, Yano M (2012) Variation in heading date conceals quantitative trait loci for other traits of importance in breeding selection of rice. Breed Sci 62(3):223-234

Hu SK, Su Y, Ye WJ, Guo LB (2012) Advances in genetic analysis and molecular regulation mechanism of heading date in rice. Chin J Rice Sci 26(3):373-382

Huang XZ, Qian Q, Liu ZB, Sun HY, He SY, Luo D, Xia GM, Chu CC, Li JY, Fu XD (2009) Natural variation at the DEP1 locus enhances grain yield in rice. Nature Genet 41(4):494-497 
Ikeda K, Ito M, Nagasawa N, Kyozuka J, Nagato Y (2007) Rice ABERRANT PANICLE ORGANIZATION 1, encoding an F-box protein, regulates meristem fate. Plant J 51(6):1030-1040

Izawa T (2007) Adaptation of flowering-time by natural and artificial selection in Arabidopsis and rice. J Exp Bot 58:3091-3097

Jiao YQ, Wang YH, Xue DW, Wang J, Yan MX, Liu GF, Dong GJ, Zeng DL, Lu Z Zhu XD, Qian Q, Li JY (2010) Regulation of OsSPL14 by OsmiR156 defines ideal plant architecture in rice. Nat Genet 42(6):541-544

Kojima S, Takahashi Y, Kobayashi Y, Monna L, Sasaki T, Araki T, Yano M (2002) $\mathrm{Hd} 3 \mathrm{a}$, a rice ortholog of the Arabidopsis FT gene, promotes transition to flowering downstream of Hd1 under short-day conditions. Plant Cell Physiol 43(10):1096-1105

Komatsu M, Maekawa M, Shimamoto K, Kyozuka J (2001) The LAX1 and FRIZZY PANICLE 2 genes determine the inflorescence architecture of rice by controlling rachis-branch and spikelet development. Dev Biol 231(2):364-373

Komatsu K, Maekawa M, Ujiie S, Satake Y, Furutani I, Okamoto H, Shimamoto K, Kyozuka J (2003) LAX and SPA: Major regulators of shoot branching in rice. Proc Natl Acad Sci U S A 100(20):11765-11770

Kozaki A, Hake S, Colasanti J (2004) The maize ID1 flowering time regulator is a zinc finger protein with novel DNA binding properties. Nucleic Acids Res 32:1710-1720

Lee $S, A n G$ (2007) Diversified mechanisms for regulating flowering time in a short-day plant rice. J Plant Biol 50:241-248

Li SB, Qian Q, Fu ZM, Zeng DL, Meng XB, Kyozuka J, Maekawa M, Zhu XD, Zhang J, Li JY, Wang YH (2009) Short paniclel encodes a putative PTR family transporter and determines rice panicle size. Plant J 58(4):592-605

Matsubara K, Yamanouchi U, Wang ZX, Minobe Y, Izawa T, Yano M (2008) Ehd2, a rice ortholog of the maize INDETERMINATE1 gene, promotes flowering by up-regulating Ehd1. Plant Physiol 148(3):1425-1435

McCouch SR, Teytelman L, Xu Y, Lobos KB, Clare K, Lincoln S (2002) Development and mapping of 2240 new SSR markers for rice (Oryza sativa L.). DNA Res 9:199-207

Oikawa T, Kyozuka J (2009) Two-step regulation of LAX PANICLE1 protein accumulation in axillary meristem formation in rice. Plant Cell 21(4):1095-1108

Ookawa T, Hobo T, Yano M, Murata K, Ando T, Miura H, Asano K, Ochiai Y, Ikeda M, Nishitani R, Ebitani T, Ozaki H, Angeles ER, Hirasawa T, Matsuoka M (2010) New approach for rice improvement using a pleiotropic QTL gene for lodging resistance and yield. Nature Commu 1:132

Park SJ, Kim SL, Lee S, Je BI, Piao HL, Park SH, Kim CM, Ryu CH, Park SH, Xuan YH, Colasanti J, An G, Han CD (2008) Rice Indeterminate 1 (Os/d1) is necessary for the expression of Ehd1 (Early heading date 1) regardless of photoperiod. Plant J 56(6):1018-1029

Qiao YL, Piao RH, Shi JX, Lee SI, Jiang WZ, Kim BK, Lee JY, Han LZ, Ma WB, Koh HJ (2011) Fine mapping and candidate gene analysis of dense and erect panicle 3, DEP3, which confers high grain yield in rice (Oryza sativa L.). Theor Appl Genet 122(7):1439-1449

Saito H, Okumoto Y, Yoshitake Y, Inoue H, Yuan Q, Teraishi M, Tsukiyama T, Nishida H, Tanisaka T (2011) Complete loss of photoperiodic response in the rice mutant line $\mathrm{X} 61$ is caused by deficiency of phytochrome chromophore biosynthesis gene. Theor Appl Genet 122(1):109-118

Tabuchi H, Zhang Y, Hattori S, Omae M, Shimizu-Sato S, Oikawa T, Qian Q, Nishimura M, Kitano H, Xie H, Fang XH, Yoshida H, Kyozuka J, Chen F, Sato Y (2011) LAX PANICLE2 of rice encodes a novel nuclear protein and regulates the formation of axillary meristems. Plant Cell 23(9):3276-3287

Takahashi Y, Shomura A, Sasaki T, Yano M (2001) Hd6, a rice quantitative trait locus involved in photoperiod sensitivity, encodes the alpha subunit of protein kinase CK2. Proc Natl Acad Sci U S A 98(14):7922-7927

Tamaki S, Matsuo S, Wong HL, Yokoi S, Shimamoto K (2007) Hd3a protein is a mobile flowering signal in rice. Science 316:1033-1036

Taoka K, Ohki I, Tsuji H, Furuita K, Hayashi K, Yanase T, Yamaguchi M, Nakashima C, Purwestri YA, Tamaki S, Ogaki Y, Shimada C, Nakagawa A, Kojima C, Shimamoto K (2011) 14-3-3 proteins act as intracellular receptors for rice Hd3a florigen. Nature 476(7360):332-335

Temnykh S, Park WD, Ayres N, Cartinhour S, Hauck N, Lipovich L, Cho YG, Ishii T, McCouch SR (2000) Mapping and genome organization of microsatellite sequences in rice. Theor Appl Genet 100:697-712

Terao T, Nagata K, Morino K, Hirose T (2010) A gene controlling the number of primary rachis branches also controls the vascular bundle formation and hence is responsible to increase the harvest index and grain yield in rice. Theor Appl Genet 120(5):875-893
Tripathi AK, Pareek A, Sopory SK, Singla-Pareek SL (2012) Narrowing down the targets for yield improvement in rice under normal and abiotic stress conditions via expression profiling of yield-related genes. Rice 5:37

Tsuji H, Tamaki S, Komiya R, Shimamoto K (2008) Florigen and the photoperiodic control of flowering in rice. Rice 1:25-35

Wang YH, Li JY (2011) Branching in rice. Curr Opin Plant Biol 14(1):94-99

Wei XJ, Xu JF, Guo HN, Jiang L, Chen SH, Yu CY, Zhou ZL, Hu PS, Zhai HQ, Wan JM (2010) DTH8 suppresses flowering in rice, influencing plant height and yield potential simultaneously. Plant Physiol 153(4):1747-1758

Wong AYM, Colasanti J (2007) Maize floral regulator INDETERMINATE1 is localized to developing leaves and is not altered by light or the sink/ source transition. J Exp Bot 58:403-414

Wu CY, You CJ, Li CH, Long T, Chen GX, Byrne ME, Zhang QF (2008) RID1, encoding a Cys2/His2-type zinc finger transcription factor, acts as a master switch from vegetative to floral development in rice. Proc Natl Acad Sci U S A 105(35):12915-12920

Xue WY, Xing YZ, Weng XY, Zhao Y, Tang WJ, Wang L, Zhou HJ, Yu SB, Xu CG, Li $\mathrm{XH}$, Zhang QF (2008) Natural variation in Ghd7 is an important regulator of heading date and yield potential in rice. Nat Genet 40(6):761-767

Yan CJ, Zhou JH, Yan S, Chen F, Yeboah M, Tang SZ, Liang GH, Gu MH (2007) Identification and characterization of a major QTL responsible for erect panicle trait in japonica rice (Oryza sativa L.). Theor Appl Genet 115(8):1093-1100

Yan WH, Wang P, Chen HX, Zhou HJ, Li QP, Wang CR, Ding ZH, Zhang YS, Yu SB, Xing YZ, Zhang QF (2011) A major QTL, Ghd8, plays pleiotropic roles in regulating grain productivity, plant height, and heading date in rice. Mol Plant 4(2):319-330

Yano M, Katayose Y, Ashikari M, Yamanouchi U, Monna L, Fuse T, Baba T, Yamamoto K, Umehara Y, Nagamura Y, Sasaki T (2000) Hd1, a major photoperiod sensitivity quantitative trait locus in rice, is closely related to the Arabidopsis flowering time gene CONSTANS. Plant Cell 12(12):2473-2484

Yano M, Kojima S, Takahashi Y, Lin HX, Sasaki T (2001) Genetic control of flowering time in rice, a short-day plant. Plant Physiol 127:1425-1429

Yoshida A, Ohmori Y, Kitano H, Taguchi-Shiobara F, Hirano HY (2012) ABERRANT SPIKELET AND PANICLE1, encoding a TOPLESS-related transcriptional co-repressor, is involved in the regulation of meristem fate in rice. Plant J 70(2):327-339

Zhu KM, Tang D, Yan CJ, Chi ZC, Yu HX, Chen JM, Liang JS, Gu MH, Cheng ZK (2010) ERECT PANICLE2 Encodes a novel protein that regulates panicle erectness in indica rice. Genetics 184(2):343-350

doi:10.1186/1939-8433-6-24

Cite this article as: Hu et al:: A point mutation in the zinc finger motif of RID1/EHD2/OsID1 protein leads to outstanding yield-related traits in japonica rice variety Wuyunjing 7. Rice 2013 6:24.

\section{Submit your manuscript to a SpringerOpen ${ }^{\circ}$ journal and benefit from:}

- Convenient online submission

- Rigorous peer review

- Immediate publication on acceptance

- Open access: articles freely available online

- High visibility within the field

- Retaining the copyright to your article

Submit your next manuscript at springeropen.com 